\title{
Capitalismo, poluição luminosa e negação do sono: Um debate relevante para o campo da Saúde Coletiva
}

\author{
Capitalism, light pollution and sleep denial: A relevant debate to the field of Collective Health \\ Capitalismo, contaminación lumínica y negación del sueño: Un debate relevante para el campo de \\ la Salud Colectiva
}

Recebido: 12/07/2021 | Revisado: 16/07/2021 | Aceito: 17/07/2021 | Publicado: 26/07/2021

Eslia Maria Nunes Pinheiro

ORCID: https://orcid.org/0000-0002-1782-4933

Federal University of Rio Grande do Norte, Brazil E-mail: eslianunes@gmail.com

Rônisson Thomas de Oliveira Silva

ORCID: https://orcid.org/0000-0002-9779-5043

Federal University of Rio Grande do Norte, Brazil E-mail: ronissont@gmail.com

Renatha Celiana da Silva Brito

ORCID: https://orcid.org/0000-0002-9712-1321

Federal University of Rio Grande do Norte, Brazil

E-mail: renathacelianaa@hotmail.com

Cecília Nogueira Valença

ORCID: https://orcid.org/0000-0003-3998-3983 Federal University of Rio Grande do Norte, Brazil E-mail: cecilia_valenca@yahoo.com.br

Jane Carla de Souza

ORCID: https://orcid.org/0000-0002-8769-4273 Federal University of Rio Grande do Norte, Brazil E-mail: jane.souza@ufrn.br

\begin{abstract}
Resumo
Objetivo: Este artigo tem a intenção de suscitar a discussão de aspectos biológicos, culturais e socioeconômicos relacionados à poluição luminosa e à negação do sono. Metodologia: Trata-se de um ensaio, no qual é realizada uma aproximação das temáticas com o campo da Saúde Coletiva, considerando a escassez de estudos na literatura sobre essa perspectiva, e o compromisso da área com a discussão dessas configurações produtivas, sociais e culturais que influenciam a saúde e qualidade de vida dos sujeitos. Resultados e Discussão: A flexibilidade e instabilidade do mercado de trabalho, além da grande quantidade de informações e produtos a serem consumidos, tornam o dia insuficiente e a noite passa a ser utilizada para a realização de atividades diversas em detrimento do sono. Destaca-se assim, um caráter compulsório de adesão aos modos de viver influenciados pelo modelo $24 / 7$ e as consequências dessa estrutura para a saúde dos indivíduos e das populações, ao passo em que é apontada a influência do mesmo sistema capitalista para a mercantilização da saúde, que retroalimenta o sistema de exploração. Conclusão: Os efeitos do modo de produção vigente e os impactos no bem-estar dos indivíduos e populações tornam essa uma discussão fulcral para o campo da Saúde Coletiva, que pode contribuir para compreensão de uma qualidade de vida além das perspectivas produtivistas/consumistas.
\end{abstract}

Palavras-chave: Cronobiologia; Ritmo Circadiano; Transtorno do sono.

\begin{abstract}
Objective: This article intends to raise the discussion of biological, cultural and socioeconomic aspects related to light pollution and sleep denial. Methodology: This is an essay, in which the themes are approached with the field of Collective Health, considering the scarcity of studies in the literature on this perspective, and the area's commitment to the discussion of these productive, social and cultural configurations that influence the health and quality of life of the subjects. Results and Discussion: The flexibility and instability of the labor market, in addition to the large amount of information and products to be consumed, make the day insufficient and the night is now used for carrying out various activities to the detriment of sleep. Thus, a compulsory character of adherence to the ways of living influenced by the 24/7 model stands out and the consequences of this structure for the health of individuals and populations, while the influence of the same capitalist system for the commodification of health, which feeds back into the operating system. Conclusion: The effects of the current mode of production and the impacts on the well-being of individuals and populations make this a key discussion for the field of Public Health, which can contribute to the understanding of a quality of life beyond the productivist/consumerist perspectives.
\end{abstract}

Keywords: Chronobiology; Circadian rhythm; Sleep disorders. 


\begin{abstract}
Resumen
Objetivo: Este artículo pretende plantear la discusión de aspectos biológicos, culturales y socioeconómicos relacionados con la contaminación lumínica y la negación del sueño. Metodología: Se trata de un ensayo, en el que se abordan los temas con el campo de la Salud Pública, considerando la escasez de estudios en la literatura sobre esta perspectiva, y el compromiso del área con la discusión de estas configuraciones productivas, sociales y culturales que inciden en la salud y la salud. calidad de vida de los sujetos. Resultados y discusión: La flexibilidad e inestabilidad del mercado laboral, además de la gran cantidad de información y productos a consumir, hacen que el día sea insuficiente y la noche se utilice ahora para realizar diversas actividades en detrimento del sueño. Así, se destaca un carácter obligatorio de adhesión a las formas de vida influenciadas por el modelo 24/7 y las consecuencias de esta estructura para la salud de los individuos y poblaciones, mientras que la influencia del mismo sistema capitalista para la mercantilización de la salud, que retroalimenta en el sistema operativo. Conclusión: Los efectos del modo de producción actual y los impactos en el bienestar de los individuos y poblaciones hacen de esta una discusión clave para el campo de la Salud Pública, que puede contribuir a la comprensión de una calidad de vida más allá de las perspectivas productivista / consumista.
\end{abstract}

Palabras clave: Cronobiologia; Ritmo Circadiano; Trastornos del sueño.

\title{
1. Introdução
}

O desenvolvimento tecnológico a partir da Revolução Industrial transformou completamente os modos de viver na sociedade, ganhando ainda mais força do século XIX para o século XX, com o advento da energia elétrica. Nesse contexto, ocorreram mudanças no ritmo de trabalho e produção, assim como na percepção coletiva do tempo, que perece estar cada vez mais acelerado, consequentemente os padrões socioeconômicos e culturais também mudaram. Mercados, farmácias, indústrias, restaurantes, igrejas, entre outros estabelecimentos têm aderido cada vez mais a um modelo ininterrupto de funcionamento, o qual funciona $24 \mathrm{~h}$ por dia, 7 dias por semana, culminando na sociedade denominada 24/7.

Cidades inteiras parecem não dormir. Ainda que dentro de suas casas, os indivíduos são bombardeados ao longo do dia e da noite com uma quantidade exaustiva de demandas de trabalho, estudo e opções virtuais de consumo de informações, coisas ou pessoas (Simabukulo et al., 2017). O acesso e utilização de mídias na lógica 24/7 e a ampliação do tempo útil desencadearam um descompasso entre a capacidade fisiológica rítmica dos seres humanos, que foi moldada pela evolução ao longo de milênios e o atual contexto de vida.

O tempo do relógio não mais condiz com o tempo do mundo - nas palavras de Crary (2014), um tempo de indiferença, que não passa mais. O autor se refere a um tempo em que não há sombras, onde não há espaço para qualquer coisa que escureça, descanse ou durma. Exemplo disso, é a ocorrência da poluição luminosa, caracterizada pela dispersão da luz artificial excessiva ou mal direcionada na atmosfera, a qual é um produto do modo de produção capitalista, que afeta negativamente tanto o ser humano quanto o meio ambiente (Silvestre, 2003).

Neste cenário, o sono surge como a última barreira natural, não colonizada pelos interesses mercadológicos, dormir torna-se um ato de resistência. No entanto, adaptações das identidades pessoais e sociais à lógica 24/7 passaram a ser realizadas, numa tentativa de reduzir as fragilidades humanas que tendem a ser ignoradas ou dissimuladas. As indústrias farmacêuticas, por exemplo, disponibilizam uma quantidade cada vez maior de medicamentos e substâncias utilizadas para modular as necessidades fisiológicas de acordo com as necessidades comerciais e sociais, seja para induzir ao sono ou reduzir a necessidade de sono e acelerar a atividade do corpo.

Do ponto de vista biológico, o sono e a vigília compõem um ritmo natural e fundamental para o funcionamento e manutenção de diversas funções vitais (Reimão, 1996; Marques \& Menna-Barreto, 2003). Além disso, este ritmo é influenciado por vários fatores externos, sendo o ciclo geofísico de dia e noite o principal modulador (Carskadon, Acebo \& Jenni, 2004; Mendoza \& Challet, 2009). Por essas razões, a exposição à luz a noite pode acarretar perturbações ou alterações no ciclo sono/vigília (CSV), o que pode comprometer significativamente o funcionamento fisiológico, cognitivo e social do indivíduo, afetando a qualidade de vida e se relacionando cada vez mais ao aumento da morbidade e mortalidade (Bittencourt et al., 2005; Souza, Mussi \& Pires, 2007; Muller \& Guimarães, 2007; Santos \& Vieira, 2018). 
Vários estudos investigam as consequências da má qualidade e comprometimento do CSV em estudantes universitários (Ribeiro et al., 2013), profissionais de saúde (Rocha \& Martino, 2010; Moreira, Marcondes \& Geremia, 2015), idosos (Monteiro \& Ceolim, 2014; Gonçalves et al., 2014) e outras populações. Cabe destacar que o sexo feminino, o aumento da idade e o trabalho em turnos são fatores de riscos para algum tipo de perturbação do sono (Rodrigues, Nina \& Matos, 2014; Mendes \& Martino, 2012) e que estes estudos são mais comuns no campo da saúde (Dahl, 1999; Ferrara \& Genaro, 2001; Taras \& Potts-Datema, 2005; Aguilar et al., 2005; Oken et al., 2006).

Em contrapartida, nota-se que os estudos que tratam da discussão da poluição luminosa são especialmente das áreas de Engenharia, Física e Astronomia e menos frequentemente na área da saúde (Sasaki et al., 2009; Barbosa et al., 2011; Gargaglioni et al., 2012). Portanto, observa-se que a problematização dessas questões numa concepção crítica em relação à saúde e sociedade ainda são incipientes no campo da Saúde Coletiva.

\section{Metodologia}

Este trabalho se caracteriza como um ensaio, com o intuito de construir, com base na literatura pertinente, uma análise sobre os aspectos biológicos, culturais e socioeconômicos relacionados à poluição luminosa e à negação do sono enquanto consequências do sistema capitalista, assim como busca defender a centralidade e integração desse tema para o campo da Saúde Coletiva.

\section{Resultados e Discussão}

\section{Poluição luminosa: cidades que não dormem, pessoas que não sonham}

Os seres humanos foram moldados em um ambiente ancestral que alternava de altas intensidades luminosas de dia para baixas à noite. Neste ambiente a alternância de claro e escuro tornou-se um sinalizador importante na organização temporal do comportamento e da fisiologia humana, como por exemplo na expressão do CSV sincronizado temporalmente com o ciclo de noite e dia, respectivamente (Applemann et al., 2013). Entretanto, com o advento da luz elétrica e a revolução tecnológica, a sociedade passou a adotar um estilo de vida que favoreceu o surgimento de uma sociedade 24/7, na qual as pessoas trabalham, dormem, comem, divertem-se a qualquer hora do dia ou da noite. Essa exposição exacerbada à luz à noite (LAN) pode causar resultados adversos à saúde, tais como, prejuízos ao sono (Lunn et al., 2017).

Barbosa e colaboradores (2011) compararam o céu noturno de uma cidade com alta poluição luminosa e outro de uma zona rural, com menos incidência de luz artificial e observaram que no primeiro, o número de estrelas identificadas no céu é 4,5 vezes menor que no segundo, devido a poluição luminosa, que torna as noites cada vez mais claras, e ressaltaram este fenômeno como "um desperdício dos recursos públicos e uma barreira à natureza".

A luz artificial invadiu a noite das cidades, no entanto, a questão vai além disso. O que observamos hoje é uma noite super iluminada (poluição luminosa) e um dia pouco iluminado, tendo em vista que grande parte dos trabalhadores e estudantes nas zonas urbanas passam longos períodos dentro de espaços fechados. Muitas atividades se dão dentro de salas de aula, escritórios, indústrias, lojas, shoppings, hospitais, entre outros, o que implica em menos contato com a luz do sol e maior contato com as luzes artificiais dos ambientes. Apesar da intensidade variável dessas luzes artificiais, esta intensidade luminosa é consideravelmente baixa em relação a luz natural do dia.

Considerando que o principal sinalizador temporal do ambiente é a alternância de claro e escuro, essa desregulação culmina numa homogeneização da experiência luminosa artificial, borrando a fronteira transicional entre dia e noite, o que pode acarretar na modificação da percepção do tempo de forma que os indivíduos, por estarem desconectados da natureza e de suas temporalidades, podem "optar" por seguir padrões de ritmicidade diária que se tornam incompatíveis com as necessidades e funcionalidades biológicas. 
No entanto, não se trata necessariamente de opção. A flexibilidade e instabilidade do mercado de trabalho, além da grande quantidade de informações e produtos a serem consumidos, tornam o dia insuficiente e a noite passa a ser utilizada para a realização de atividades diversas em detrimento do sono. Afinal, a sociedade capitalista entende que o tempo de sono deveria ser gasto para o desenvolvimento de algumas atividades economicamente produtivas.

Existe uma cultura de desvalorização do sono. Segundo Crary (2014), quem nunca ouviu a frase "passamos um terço das nossas vidas dormindo"? Essa é só uma das tantas expressões que podem ser usadas na perspectiva de caracterizar pejorativamente a fase de sono, como perda de tempo. Isso se evidencia como uma consequência do nosso estilo de vida exageradamente acelerado, em que pausas e descansos não são aceitos ou bem interpretados.

Contudo deve-se considerar que a rotina da sociedade de trabalhar, estudar, consumir e realizar cada vez mais atividades de forma a estender as horas em vigília leva a prejuízos à saúde geral do indivíduo (Frank, 2015; Rattenborg et al., 2017; Xie et al., 2013) e diminuição do desempenho cognitivo (Goel, 2017; Tononi \& Cireli, 2014; Valdez et al., 2010). Estudo realizado por Calamaro, Mason e Ratcliffe (2009) identificou o prejuízo na capacidade funcional de adolescentes ao longo do dia provocado pelo consumo de bebidas com cafeína e utilização de diferentes formas de tecnologia ao longo da madrugada.

A exposição a aparelhos eletrônicos (celulares, televisores e computadores) à noite acarreta aumento no alerta fisiológico no momento da exposição e dificulta o relaxamento necessário para dormir (Higuchi et al., 2003). Durante o uso destes eletrônicos ocorre supressão da melatonina, que é o hormônio do escuro e atua como indutor do sono em humanos, portanto, a supressão irá dificultar ainda mais que o indivíduo adormeça (Van De Lely et al., 2015). Esta mesma exposição atrasa não só o sono na noite do uso dos aparelhos eletrônico, mas também na noite seguinte (Chang et al., 2014). O impacto dessa modulação da luz pode ser maior ou menor de acordo com o comprimento de onda ao qual o indivíduo está exposto (Badia et al., 1991).

A dificuldade em iniciar o sono somada a horários matinais de estudo e trabalho podem acarretar privação de sono e mudanças na ritmicidade diária do organismo, que comprometem inúmeros aspectos fisiológicos do organismo (Crowey et al., 2015). Independentemente da idade, as alterações no chamado ciclo sono-vigília podem aumentar o risco de acidentes fatais no trabalho e problemas somáticos e psicológicos (câncer; afecções cardíacas, gastrintestinais e reprodutivas; sofrimentos psíquicos) (Santos et al, 2014). Desta forma, para o organismo se manter em equilíbrio e saudável é necessário manter certa regularidade nos horários de dormir e acordar, e uma duração considerável que varia de indivíduo para indivíduo (Gomes et al., 2010).

\section{Os fins do sono, as reformas neoliberais e o campo da Saúde Coletiva}

No contexto atual de iniquidades sociais e modernidade tecnológica, o humano vive um constante desafio temporal entre a velocidade das mudanças do ambiente e a capacidade de adaptação fisiológica do organismo para manter a saúde. É comum que as queixas dos indivíduos sejam encaixadas em diagnósticos e prontamente medicalizadas (Santos et al., 2014). Por exemplo, episódios de insônia e sonolência diurna podem levar ao abuso de substâncias relaxantes e estimulantes, respectivamente, tais como: os sedativos/hipnóticos e as anfetaminas/metanfetaminas, substâncias que são usadas em abuso e acarretam uma série de prejuízos para a saúde a médio e longo prazo, como dependência, overdose e morte (Lipari, Williams \& Horn, 2017). Portanto, o nó crítico desta questão é como encarar e encontrar uma forma de sobreviver ao dilema de um modelo capitalista de produção ininterrupta, o qual se sobrepõe à saúde e qualidade de vida das pessoas.

O modelo de Atenção à Saúde hegemônico no Brasil apresenta traços fundamentais, como a medicalização dos problemas, privilégio da medicina curativa e o processo saúde-doença como mercadoria, dando ênfase ao "biologismo" (Paim, 2008). Para cada agravo, uma solução encapsulada estar à espera na farmácia mais próxima. Dessa forma, esse modelo 
fortalece o sistema capitalista e gera lucros a partir das necessidades provocadas pelo seu próprio funcionamento.

Os elementos constitutivos das práticas de saúde se adequam conforme as relações sociais se realizam historicamente (Merhy, 1987). Dito isso, sabe-se que essas práticas são sociais e estruturadas de acordo com o movimento infra e supraestrutural do capital, que imprime o seu sentido e as suas finalidades. As atividades sanitárias atuais tomam, portanto, como seu objeto os grupos enquanto classes sociais e o meio ambiente enquanto lugar de produção do capital e de reprodução dessas classes. De acordo com Menezes, Moretti e Reis (2019), desde 2016 o neoliberalismo no Brasil tem assumido uma face na qual o Estado não mais se autolimita e zela a concorrência de mercado, mas sim um mercado que ativamente intervém avaliando e regulando as ações do governo, considerando critérios estritamente econômicos.

Esses governos neoliberais desfinanciam as políticas públicas, incluindo a saúde, e apoiam a chamada "reestruturação produtiva". Segundo Martins e Molinaro (2013), este modelo de desenvolvimento econômico que rompe com os pressupostos do direito trabalhista, no Brasil ganha proporções maiores considerando as carências sociais fazendo com o que a economia cresça mais do que o ingresso da população economicamente ativa, o que desencadeia desigualdade social, marginalização dos sujeitos com menor escolaridade, a exploração da mão de obra e, segundo os autores, faz com que a modernidade chegue de forma perversa para a maior parte da população.

Partindo dessas premissas é possível dizer que essas condições, afetam de algumas maneiras a relação dos sujeitos com o trabalho, as cidades e o sono: 1) precarizando os vínculos empregatícios e aumentando o empobrecimento da população, o que favorece a exploração da mão de obra e a sujeição a regimes de trabalho que não respeitam as necessidades psíquicas e fisiológicas de descanso e sono; 2) desmontando o serviço público de saúde em detrimento do favorecimento do setor privado, o que contribui para a mercantilização da saúde, reduzindo a participação do Estado na oferta de bens e serviços de qualidade à população, preservando e ampliando os ganhos dos mais ricos e retroalimentando o processo de consumo e trabalho 24/7 (Santos \& Vieira, 2018).

Cabe fazer referência à noção de determinação social saúde, que busca compreender a saúde a partir de uma perspectiva dialética - na qual os processos sociais são considerados e analisados como processos e não como fatores isolados, com ênfase nas relações de trabalho/produção (Garbois, Sodré \& Dalbello-Araujo, 2017).

No interior de uma relação indissociável entre aspectos biológicos e sociais (nestes, inclua-se aí aspectos culturais, políticos etc.), a saúde humana ganha seus contornos individual e coletivamente, transitando em vários níveis e de diversas formas, dependendo das relações sociais estabelecidas, em especial as relações sociais de produção (Souza, 2020, p. 176).

Diante disto, ressalta-se a importância da Saúde Coletiva como um campo científico e social bastante amplo teórica e metodologicamente, que surgiu no Brasil no auge do movimento de luta pelo fim da ditadura na década de 1970 e no berço da construção dos ideais sanitaristas que culminaram na criação do Sistema Único de Saúde (SUS). Esta área tem suas raízes no preventivismo e na medicina social, fazendo oposição à fragmentação das práticas de cuidado em saúde, à centralidade do saber médico e trazendo aspectos sociais e econômicos para o debate da saúde (Osmo \& Schraiber, 2015).

Além disso, a Saúde Coletiva se configura como um campo de práticas que pauta processos político-pedagógicos, suscitando o "desenvolvimento de um pensar crítico e reflexivo, permitindo desvelar a realidade e propor ações transformadoras que levem o indivíduo à sua autonomia e emancipação como sujeito histórico e social, capaz de propor e opinar nas decisões de saúde para cuidar de si, de sua família e de sua coletividade" (Falkenberg, 2014, p. 848). Portanto, os estudos em Saúde Coletiva devem apresentar um compromisso com a análise da relação entre as políticas públicas e as configurações produtivas, sociais e culturais que influenciam a qualidade de vida dos sujeitos.

Assim, defendemos a importância de pautar o debate acerca do modelo 24/7, bem como suas consequências como a 
poluição luminosa e a negação do sono, na discussão do campo da Saúde Coletiva, contribuindo assim com o debate de que as práticas em saúde não reproduzam essa lógica de reprodução e adoecimento, mas ao contrário, favoreçam a promoção do bemestar e do desenvolvimento social adequado.

\section{Considerações Finais}

Atualmente temos uma sociedade que busca se adequar ao sistema econômico dominante e não um sistema que se adequa aos seres vivos e ao planeta como um todo. A lógica capitalista de produção e consumo ininterruptos (24/7) provoca uma desconexão entre o ritmo biológico do ser humano e o ritmo da natureza através da criação de uma natureza artificial, sem distinção entre dia e noite. Soma-se a isso a demanda e a oferta inesgotáveis por produção e consumo de produtos, pessoas, experiências e tecnologias visuais e sonoras. A poluição luminosa e a negação do sono surgem como consequências desse modelo e estão diretamente relacionadas à vulnerabilidade biopsíquica e social das pessoas vivendo na sociedade contemporânea. Essa imbricação entre os efeitos do modo de produção vigente e o bem-estar dos indivíduos e das populações tornam essa uma discussão fulcral para o campo da Saúde Coletiva, que pode contribuir para compreensão de uma qualidade de vida além das perspectivas produtivistas e consumistas. Além de trazer à tona a necessidade de repensar as relações de trabalho e educação com vistas a assumir o compromisso ético-político do campo para com a coletividade e construir alternativas emancipadoras em saúde.

\section{Referências}

Aguilar, F. S. (2005). Hábitos de sueño y problemas relacionados con el sueño en adolescentes: relación con el rendimiento escolar. Aten Primaria. 35, 408414.

Appleman, K., Kenneth, A. L., Mariana, G. F. \&, Mark, S. R. (2013). Controlling light-dark exposure patterns rather than sleep schedules determines circadian phase. Sleep Medicine. 14, 456-461.

Badia, P., Myers, B., Boecker, M., Culpepper, J. \& Harsh J. R. (1991). Bright light effects on body temperature, alertness, EEG and behavior. Physiology e Behavior. 50 (3), 583-588.

Barbosa, J. L. E., Beduschi Filho, L. F. S., Morais, L. F. S. \& Defavari, R. (2011). Novas Abordagens Sobre a Poluição Luminosa No Céu Noturno. Revista Ciências do Ambiente On-Line. 6 (2).

Benedito-Silva, A.A. (2008). In: Tufok, S. Cronobiologia do ciclo vigília-sono (285-298). Handbook of behavioral neurobiology-biological rhythms.

Bittencourt, L. R. A., Silva, R. S., Santos, R. F., Pires, M. L. N. \& Mello, M. T. (2005). Sonolência excessiva. Rev Bras Psiquiatr. 27 (1), $16-21$.

Brasil. (2000). Constituição da República Federativa do Brasil. Ed. Revista dos Tribunais.

Calamaro, C. J., Mason, T. B. A. \& Ratcliffe, S. J. (2009). Adolescents living the 24/7 lifestyle: effects of caffeine and technology on sleep duration and daytime functioning. Pediatrics. 123 (6), 1005-1010.

Carskadon, M. A., Acebo, C. \& Jenni, O. G. (2004). Regulation of Adolescent Sleep: Implications for Behavior. Annals Of The New York Academy Of Sciences. 1021 (1), 276-291.

Carvalho, T. M. C. S., Silva Junior, I. I., Siqueira, P. P. S. Almeida, J. O. Soares, A. F. \& Lima, A. M. J. (2013). Qualidade do sono e sonolência diurna entre estudantes universitários de diferentes áreas. Revista Neurociências. 21 (3), 383-387.

Chang, A. M., Aeschbach, D., Duffy, J. F. \& Czeisler, C.A. (2015). Evening use of light-emitting ereaders negatively affects sleep, circadian timing, and nextmorning alertness. Proceedings of the National Academy of Sciences. 112 (4), 1232-1237.

Crowley, S. J., Cain, S. W., Burns, A. B., Acebo, C. \& Carskadon, M.A. (2015). Increased Sensitivity of the circadian system to light in early/mid-puberty. The Journal of clinical endocrinology and metabolism. 100 (11), 4067-4073.

Dahl, R. E. (1999). The consequences of insufficient sleep for adolescents: links between sleep and emotional regulation. In: Wahlstrom, K.L. (ed) Adolescent sleep needs and school starting time. Bloomington, Phi Delta Kappa Education Foundation. 29-44.

Falkenberg, M. B., Mendes, T. P. L., Moraes, E. P. \& Souza, E. M. (2014). Educação em saúde e educação na saúde: conceitos e implicações para a saúde coletiva. Ciência \& Saúde Coletiva [online]. 19 (3), 847-852.

Ferrara, M. \& Gennaro, L. (2001). How much sleep do we need? Sleep Med Rev. 5, 155-179. 
Frank, M. G. (2015). Sleep and synaptic plasticity in the developing and adult brain. Current Topics in Behavioral Neurosciences. https://doi.org/10.1007/7854_2014_305

Garbois, J. A., Sodré, F. \& Dalbello-Araujo, M. (2017). Da noção de determinação social à de determinantes sociais da saúde. Saúde em Debate [online]. 41 (112), 63-76.

Galeano, (1987). As Veias abertas da América Latina. tradução de Galeno de Freitas. Rio de Janeiro: Editora Paz e Terra.

Gargaglioni, S. R., Dupas, F. A. \& Rodriguez-Ardila, A. (2012). Previsão dos Impactos Causados por Poluição Luminosa com Ênfase nos Sítios de Observação Astronómica e Síntese da Proposta de Legislação Nacional. Holos Environment. 12 (1), 27-40.

Goel, N. (2017). Neurobehavioral Effects and Biomarkers of Sleep Loss in Healthy Adults. Current Neurology and Neuroscience Reports. 17 (11). https://doi.org/10.1007/s11910-017-0799-x

Gomes, M. M., Quinhones, M. S., Engelhardt, E. (2010). Neurofisiologia do sono e aspectos farmacoterapêuticos dos seus transtornos. Revista Brasileira de Neurologia. 46, 05-15.

Gonçalves, D., Altermann, C., Vieira, A., Machado, A. P., Fernandes, R., Oliveira, A. \& Mello-Carpe, P. B. (2014). Avaliação das funções cognitivas, qualidade de sono, tempo de reação e risco de quedas em idosos institucionalizados. Estudos Interdisciplinares sobre o envelhecimento. 19 (1).

Higuchi, S., Motohashi, Y. Liu, Y., Ahara, M. \& Kaneko, Y. (2003). "Effects of VDT Tasks with a Bright Display at Night on Melatonin, Core Temperature, Heart Rate, e Sleepiness." Journal of Applied Physiology. 94, 1773-1776.

Lipari, R. N., Williams, M., \& Horn, S. L. V. (2017). Why Do Adults Misuse Prescription Drugs? The CBHSQ Report. Center for Behavioral Health Statistics and Quality, Substance Abuse and Mental Health Services Administration, Rockville, MD.

Lunn, R. M., Blask,D. E., Coogan, A. N., Figueiro, M. G., Gorman, M. R. G., Hall, J. E., Hansen, J., Nelson, R. J., Panda, R., Smolensky, M. H., Stevens, R. G., Turek, F. W., Vermeulen, R., Carreón, T., Caruso, C. C., Lawson, C. C., Thayer, K. A., Twery, M. J., Ewens, A. D., Garner, S. C., Schwingl, P. J. \& Boyd, W. A., (2017). Health consequences of electric lighting practices in the modern world: A report on the National Toxicology Program's workshop on shift work at night, artificial light at night, and circadian disruption. Ciência do Ambiente Total. 607, 1073-1084.

Marques, M. \& Menna-Barreto, L. (2003). Cronobiologia: Principios e Aplicações. Fiocruz.

Martins, M.I.C. \& Molinaro, A. (2013). Reestruturação produtiva e seu impacto nas relações de trabalho nos serviços públicos de saúde no Brasil. Ciência \& Saúde Coletiva. 18 (6), 1667-1676.

Medeiros, A. L. D., Lima, P. F., Almondes, K. M., Dias Junior, A. S., Rolim, S. A. M. \& Araújo, J. F. (2002). Hábitos de sono e desempenho em estudantes de medicina. Revista Saúde do centro de ciências da saúde (UFRN). 16 (1), 49-54.

Mendes, S. S. \& Martino, M. M. F. (2012). Trabalho em turnos: estado geral de saúde relacionado ao sono em trabalhadores de enfermagem. Rev Esc Enferm. USP. 46 (6), 1471-1476.

Mendoza, J. \& Challet, E. (2009). Brain clocks: From the Suprachiasmatic Nuclei to a cerebral network. The Neuroscientist. 15 (5), 477-488.

Menezes, A. P. R., Moretti, B. \& Reis, A. A. C. (2009). O futuro do SUS: impactos das reformas neoliberais na saúde pública - austeridade versus universalidade. Saúde em Debate [online]. 43 (5), 58-70.

Merhy, E. E. (2014). Capitalismo e a saúde pública: a emergência das práticas sanitárias no Estado de Säo Paulo. Rede UNIDA.

Monteiro, N. T. \& Ceolim, M.F. Qualidade do sono de idosos no domicílio e na hospitalização. Texto \& Contexto Enfermagem. 23 (2), 356-364.

Moreira, M. M., Marcondes, C. \& Geremia, D. S. (2015). Padrões de sono entre os profissionais de enfermagem. Revista de Atenção à Saúde. 13 (44), 11-16.

Muller, M. R. \& Guimarães, S. S. (2007). Impacto dos transtornos do sono sobre o funcionamento diário e a qualidade de vida. Estud Psicol. 24 (4), $519-528$.

Osmo, A. \& Schraiber, L. B. (2015). O campo da Saúde Coletiva no Brasil: definições e debates em sua constituição. Saúde Soc. 24, 205-218.

Oken, B. S., Salinsky, M. C. \& Elsas, S.M. (2006). Vigilance, alertness, or sustained attention: physiological basis and measurement. Clinical neurophysiology. 117 (9), 1885-1901.

Paim, J. S. (2008). Modelos de atenção à saúde no Brasil. In: Política e sistema de saúde no Brasil. 547-573.

Ramos, M. N. (2006). A pedagogia das competências: autonomia ou adaptação? Cortez.

Rattenborg, N. C., La Iglesia, H. O., Kempenaers, B., Lesku, J. A., Meerlo, P. \& Scriba, M. F. (2017). Sleep research goes wild: New methods and approaches to investigate the ecology, evolution and functions of sleep. Philosophical Transactions of the Royal Society B: Biological Sciences. https://doi.org/10.1098/rstb.2016.0251

Reimão, R. (1996). Sono: estudo abrangente. Atheneu.

Ribeiro, C. R. F., Silva, Y. M. G. P., Oliveira, S. M. C. (2014). O impacto da qualidade do sono na formação médica. Rev soc bras clín méd. 12 (1), 8-14.

Rocha, M. C. P. \& Martino, M. M. F. (2010). O estresse e qualidade de sono do enfermeiro nos diferentes turnos hospitalares. Revista da Escola de Enfermagem da USP. 44 (2), 280-286.

Rodrigues, M., Nina, S. \& Matos, L. (2014). Como dormimos? - Avaliação da qualidade do sono em cuidados de saúde primários. Revista Portuguesa de Medicina Geral e Familiar. 30 (1), 16-22. 
Santos, A. F., Mussi, F. C., Pires, C. G. S. (2007). Qualidade do sono e fatores associados em universitários de enfermagem. Acta Paulista de Enfermagem.33.

Santos, I. S. \& Vieira, F. S. (2018). Direito à saúde e austeridade fiscal: o caso brasileiro em perspectiva internacional. Ciência \& Saúde Coletiva. 23, 23032314.

Santos, L. C., Castro, N. J., Ruback, O. R., Trigo, T.J. B., Rocha, P. M. B. (2014). Transtornos do ciclo sono-vigilia/circadiano-Uma revisão de literatura. Brazilian Journal of Surgery and Clinical Research. 7 (2), 38-43.

Sasaki, A. T., Devidis, B. M. N., Morigaki, C. A., Tozoni, D. C. (2009). Análise da poluição luminosa e a visibilidade noturna do céu. Revista Ciências do Ambiente On-Line. 5 (2), 1-6.

Silva, C. B. L. (2017). Estudo da Eficiência Luminosa e Energética do Sistema de Iluminação Pública da Cidade do Porto. 139 f. Dissertação (Mestrado em Engenharia Electrotécnica e de Computadores - Área de especialização de Sistemas de Energia) - Faculdade de Engenharia do Porto. Cidade do Porto, out/2007. Disponível em: https://repositorio-aberto.up.pt/bitstream/10216/12295/2/Texto\%20integral.pdf. Acesso em: 19 de novembro de 2019.

Silvestre, R. F. (2003). Campanha contra a poluição luminosa. Revista Macrocosmo. 1.

Simabukulo, L. A. N., Correa, L. F. S., Santos, M. M. O. \& Martins, M. (2017). Energia, Industrialização e Modernidade. História Social. Projeto ELETROMEMÓRIA - História da energia elétrica no estado de São Paulo. Universidade de São Paulo (USP).

Siqueira-Batista, R. \& Batista, R. S. (2009). Os anéis da serpente:a aprendizagem baseada em problemas e as sociedades de controle. Cien Saude Colet. 14 (4), 1183-1192

Siqueira-Batista, R., Gomes, A. P. \& Albuquerque, V. S. (2013). Educação e competências para o SUS: é possível pensar alternativas à (s) lógica (s) do capitalismo tardio?. Ciência \& Saúde Coletiva. 18, 159-170.

Souza, D. O. (2020). O caráter ontológico da determinação social da saúde. Serviço Social \& Sociedade [online]. 137, 174-191.

Souza, J. C., Souza, N. M., Ashiro, E. S. H. \& Schaedler, R. (2007). Sonolência diurna excessiva em pré-vestibulandos. J Bras Psiquiatr. 56 (3), $184-187$.

Souza, J. C., Guimarães, L. A. M. (1999). Insônia e qualidade de vida. Campo Grande MS: Universidade Católica Dom Bosco.

Taras, H. \& Potts-Datema, W. (2005). Sleep and student performance at school. J Sch Health. 75, 248- 254.

Tononi, G. \& Cirelli, C. (2014). Sleep and the Price of Plasticity: From Synaptic and Cellular Homeostasis to Memory Consolidation and Integration. Neuron. https://doi.org/10.1016/j.neuron.2013.12.025

Valdez, P. Ramirez, C., Garcia, A., Talamantes, J. \& Cortez, J. (2010). Circadian and homeostatic variation in sustained attention. Chronobiology International. https://doi.org/10.3109/07420521003765861

Valle, R. (2003). O conhecimento em ação: novas competências para o trabalho no contexto da reestruturação produtiva. Rio de Janeiro: Relume Dumará.

Van Der Lely, S., Frey, S., Garbazza, C., Wirz-Justice, A., Jenni, O. G., Steiner, R., Wolf, S., Cajochen, C., Bromundt V. \& Schimidt, C. (2015). Blue Blocker Glasses as a Countermeasure for Alerting Effects of Evening Light-Emitting Diode Screen Exposure in Male Teenagers. Journal of Adolescent Health. 56 (1), 113-119.

Xie, L., Hongyi, K., Qiwu, X., Michael, J. C., Yonghong, L., Meenakshisundaram, T., John, O., Daniel, J. C., Charles, N., Jeffrey, J., Takahiro, T., Rashid, D. \& Maiken, N. (2013). Sleep drives metabolite clearance from the adult brain. Science. https://doi.org/10.1126/science.1241224 